\title{
Estudos do Campo Geotérmico Raso em Jenipapo de Minas: Implicações para escolha de sítios para barragens subterrâneas na região de "polígonos de seca".
}

\author{
Fábio P. Vieira, Jorge L.S. Gomes e Valiya M. Hamza, Observatório Nacional - ON/MCT, Rio de Janeiro.
}

Copyright 2016, SBGf - Sociedade Brasileira de Geofísica

Este texto foi preparado para a apresentação no VII Simpósio Brasileiro de Geofísica, Ouro Preto, 25 a 27 de outubro de 2016. Seu conteúdo foi revisado pelo Comitê Ouro Preto, 25 a 27 de outubro de 2016. Seu conteúdo foi revisado pelo Comitê
Técnico do VII SimBGf, mas não necessariamente representa a opinião da SBGf ou de seus associados. É proibida a reprodução total ou parcial deste material para propósitos comerciais sem prévia autorização da SBGf.

\begin{abstract}
Temperature profiles of four shallow wells in the region of Jenipapo de Minas (MG) were examined for identifying thermal signals of subsurface fluid flows. The analysis was carried out based on fits of observational data to onedimensional models of geothermal disturbances in shallow wells. The results are compatible with indications of down flow of groundwater, with velocities of the order of $10^{-7} \mathrm{~m} / \mathrm{s}$. However, such effects are superimposed on perturbations induced by changes in surface temperature of 2 to $3^{\circ} \mathrm{C}$ in the last 50 years. We discuss the implications of these results in the selection of sites for construction of underground dams, a solution considered highly suitable for securing water supply in regions with frequent episodes of drought.
\end{abstract}

\section{Introdução}

As regiões que sofrem com secas no Estado de Minas Gerais possuem uma área estimada em $103.590 \mathrm{~km}^{2}$ distribuídas em 85 municípios, e que representa 10,54\% da área do Semiárido Brasileiro. Os Vales do Jequitinhonha e Mucuri se encontram como regiões com precipitações pluviométricas escassas, além de elevada taxa de evaporação regional.

Uma das formas de minimizar problemas de seca seria captação de águas subterrâneas. Contudo, tentativas de empregar os métodos convencionais, baseados em perfurações de poços, revelaram ser pouco eficiente em terrenos de baixa porosidade e permeabilidade. No entanto, os fluxos de águas subterrâneas nas camadas rasas do solo podem ser capturados com construção de barragens subterrâneas. A maior dificuldade neste método é a escolha de sítios apropriados, onde ocorrem movimentos descendentes de água subterrânea. Neste contexto, os métodos de geotermia rasa se destacam como um dos meios de avaliação complementar de baixo custo para avaliação de infiltrações de águas pluviais em camadas de solos (Hamza, 1982).

\section{Princípio do Método de Geotermia rasa}

O campo térmico do subsolo, em profundidades de até algumas dezenas de metros, sofre efeito das variações diurnas e sazonais das temperaturas da superfície. Em profundidades maiores as temperaturas são menos afetadas pelas variações de curta duração que ocorrem na superfície, e aumentam linearmente com a profundidade devido ao fluxo de calor proveniente do interior da Terra. O valor médio do gradiente geotérmico para o Estado de Minas Gerais é de 15 a $25^{\circ} \mathrm{C} / \mathrm{km}$. Contudo, os movimentos de água subterrânea através das camadas permeáveis ou do sistema de fraturas podem induzir modificações locais na distribuição de temperaturas determinada pelo gradiente geotérmico. Geralmente fluxos de água subterrânea nas formações geológicas permeáveis induzem alterações no campo térmico em escala regional. Em alguns casos específicos perfurações também podem propiciar fluxo de água no interior de poços, induzindo desta forma alterações localizadas no campo térmico. A figura 1 ilustra a localização da área de estudo.

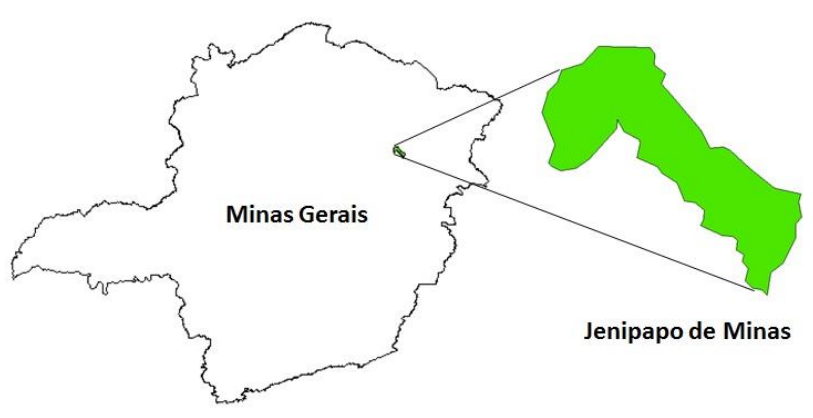

Figura 1 - Localização da área de estudo

\section{Contexto Hidrogeológico}

No município de Jenipapo podem-se distinguir dois domínios hidrogeológicos: os de rochas metamórficas do Neoproterozóico e granito do Paleozóico em locais de afloramentos de embasamento; e os de coberturas detríticas do Cenozóico, sobrejacentes ao embasamento. Esses domínios são geralmente enquadrados nos sistemas aquíferos granular e fissurados.

O sistema aquífero granular é representado por sedimentos pouco consolidados que constituem as coberturas detríticas de composição arena-argilosa. Em termos hidrogeológicos possuem porosidade primária e boa permeabilidade. São aquíferos potencialmente fracos, mas importantes no processo de recarga dos aquíferos fissurais subjacentes, através de filtração vertical. Já o sistema aquífero fissurado ocorre em toda extensão do município estando relacionado às rochas xistosas.

De um modo geral, apresenta baixo potencial hidrogeológico, sendo as feições estruturais responsáveis por variações locais de produtividade. Nesse sistema predominam as fraturas, falhas e xistosidades que fornecem a porosidade secundária $O$ potencial hidrogeológico é dependente da densidade e intercomunicação das descontinuidades, aspecto que 
geralmente se traduz em reservatórios aleatórios e de pequena extensão (CPRM 2005). Apresenta-se na Figura-2 o mapa da hidrogeologia simplificada.

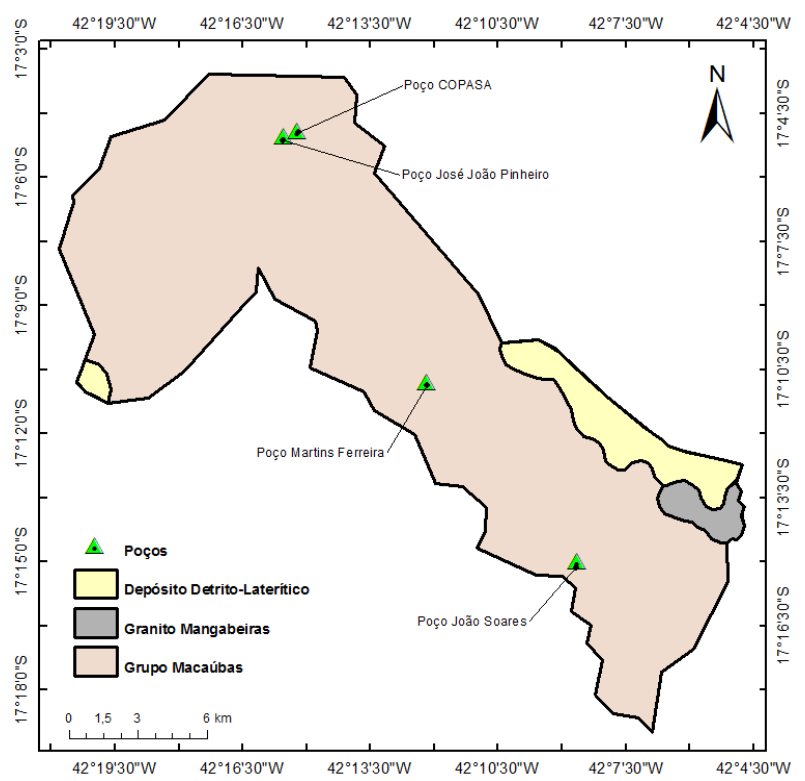

Figura 2 - Geologia simplificada do município de Jenipapo de Minas - CPRM 2005 (Adaptado Vieira 2016).

\section{Perfilagens Térmicas}

Foram efetuados perfilagens térmicas em quatro poços na área deste estudo. Todos os poços se encontram distribuídos nas regiões do Grupo Macaúbas conforme ilustrado na figura 2. De modo geral, os perfis térmicos apresentaram características semelhantes entre si. Os perfis parecem ser constituídos de dois segmentos distintos, designados aqui como superior e inferior. $O$ segmento superior, desde a superfície até cerca de 20 metros de profundidade, sofre influência direta das variações de temperatura do ar na superfície. Os segmentos inferiores dos perfis térmicos, situados entre cerca de 20 e 120 metros, são caracterizados por variações quase desprezíveis de temperatura. Nos perfis as temperaturas destes segmentos são praticamente constantes, apresentando indícios de movimentos de água nesses intervalos. A figura 3 ilustra as perfilagens térmicas nos poços de João Soares, COPASA, Martins Farinheira e José João Pinheiro.

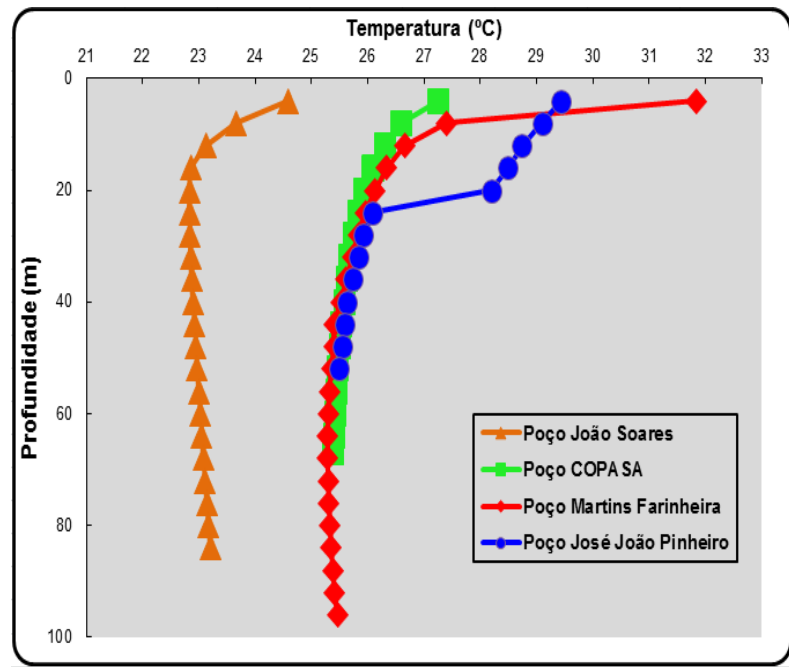

Figura 3-Perfilagens térmicas nos poços de João Soares, COPASA, Martins Farinheira e José João Pinheiro.

Note que todos os poços apresentam uma zona de temperatura constante abaixo de 20 metros de profundidade, indicando movimentação de água dentro do poço.

É importante destacar que não há linearidade na distribuição vertical de temperaturas em poços rasos, por ser oriundo de atuação de dois processos de transporte e redistribuição de calor nas camadas subsuperfíciais. O primeiro deles o movimento de águas e o segundo as mudanças climáticas. A interpretação de perfis rasos geralmente torna-se difícil na presença de efeitos compostos dessas duas perturbações. Nesses casos foram realizadas reconstruções das perturbações de forma separada para os quatros poços perfilados.

\section{Identificação de movimento de águas subterrâneas}

A abordagem em relação ao movimento de águas se baseia na solução da equação diferencial para transferência simultânea de calor por condução e por convecção. O movimento de infiltração de águas significa transporte advectivo de calor sobreposto ao fluxo condutivo de calor. A figura 4 ilustra a distribuição de temperaturas, que depende do sentido de movimento: ascendente (curva convexa) ou descendente (curva côncava). 


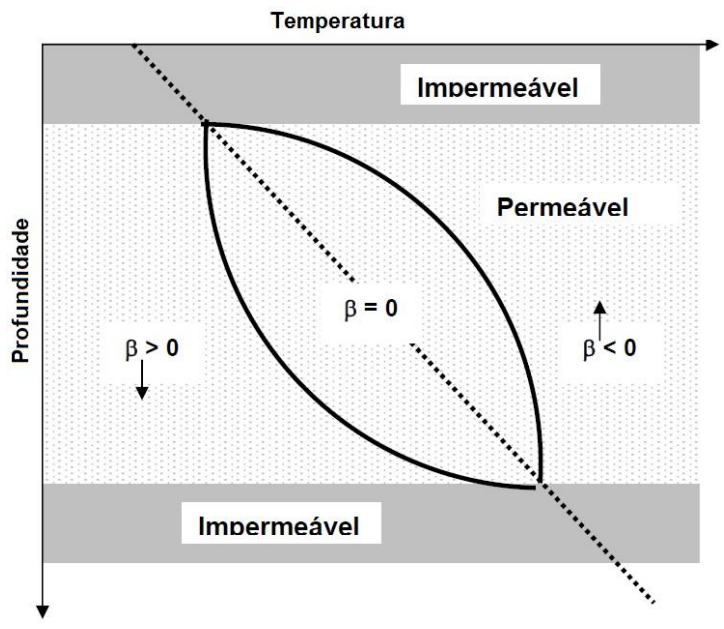

Figura 4 - Representação esquemática da distribuição de temperaturas numa camada permeável na presença de movimento vertical de fluidos

O princípio geral do método geotérmico para o estudo dos fluxos de água subterrânea pode ser compreendido considerando o papel de transferência de calor em regime advectivo de camadas condutora do subsolo. Em ambientes tectônicos relativamente estáveis mudanças no regime térmico ocorrem em escalas de tempo que são grandes em comparação com escalas de tempo passíveis de medidas experimentais.

Segundo Bredehoeft e Papadopulos (1965), o campo de temepraturas nos intervalos de movimento de águas subterraneas é determnado pela equação:

$$
\frac{T_{z}-T_{0}}{T_{L}-T_{0}}=\left[\frac{\exp \cdot(\beta \cdot z / L)-1}{\exp \cdot(\beta)-1}\right]
$$

O lado esquerdo da equação (1) é a temperatura adimensional $(\theta)$ cuja distribuição vertical depende do sinal do parâmetro $\beta$, que pode ser positivo ou negativo dependendo do sentido de movimento de água, com velocidade $V_{z}$. Assim, um grafico da profundidade adimensional ( $\mathrm{z} / \mathrm{L})$ em função da temperatura adimensional $(\theta)$ deve revelar o efeito de transferência de calor por fluxo de água vertical. $O$ parametro $\beta$ é dado pela relacao:

$$
\beta=\frac{\rho_{f} \cdot C_{f} \cdot v_{f} \cdot L}{\lambda_{S}}
$$

Nota-se que parâmetro $\beta$ representa a razão entre as parcelas de energia térmica transportada respectivamente por advecção e condução, ou seja, o número de Peclet.

\section{Efeitos térmicos de mudanças climáticas}

Variações na temperatura da superfície decorrente de mudanças climáticas geram perturbações nos perfis térmicos de poços rasos. Aumentos na temperatura da superfície (aquecimento climático) geram concavidades nos perfis térmicos, conforme ilustrado na figura 5 abaixo (faixa em cor vermelha).

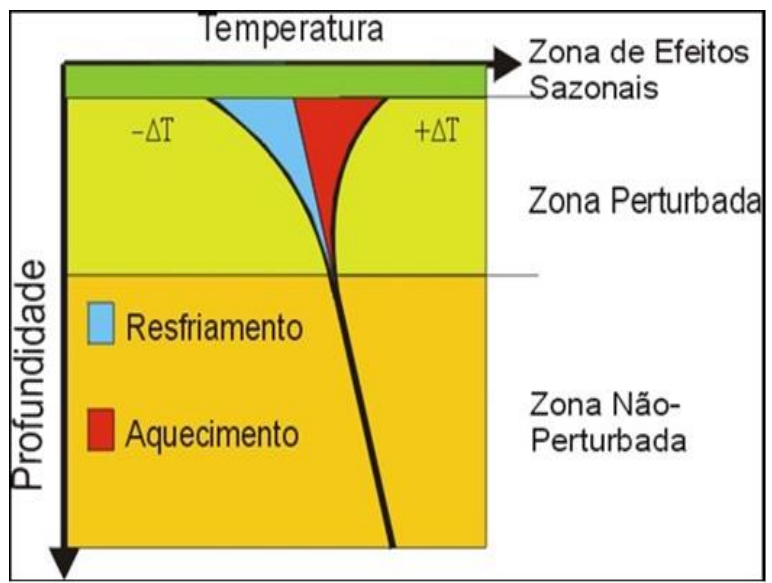

Figura 5 - Representação esquemática de perfil térmico na presença de perturbações climáticas

Tratando de perturbações térmicas induzidas em profundidades pequenas (em relação às dimensões da área afetada por variações climáticas), consideramos o meio semi-infinito como representativo da geometria do problema. No presente trabalho foi adotada a mudança linear para o cálculo da perturbação climática conforme listrado abaixo:

$T=T_{m} \cdot\left[\left(1+2 \cdot \frac{z^{2}}{4 k t}\right) \cdot \operatorname{erfc} \cdot\left(\frac{z}{\sqrt{4 k t}}\right)-\frac{2}{\sqrt{\pi}} \cdot\left(\frac{z}{\sqrt{4 k t}}\right) \cdot e^{\frac{-z^{2}}{4 k t}}\right]$

onde $T$ é a temperatura, $z$ a profundidade, $t$ o tempo, $\mathrm{k}$ a difusividade térmica e $T_{m}$ a temperatura não perturbada do meio.

\section{Separação dos efeitos de infiltração pluvial e de clima}

Distribuições verticais de temperaturas em camadas rasas possuem componentes de transporte de calor oriundos de movimentos de fluidos subterrâneos (processo de advecção) e difusão condutiva das perturbações induzidas por mudanças climáticas recentes. O procedimento de separação das perturbações se baseia na observação de que a magnitude de perturbação climática é relativamente maior na parte superior dos poços. Na parte inferior dos poços predominam-se efeitos de transporte advectivo de calor. O procedimento de interpretação se baseia no ajuste de dados observados na parte inferior dos poços aos valores estimados pelo modelo selecionado de perturbação hidrológica. Na parte superior de poços os valores calculados com base no modelo hidrológico são utilizados para corrigir os valores de temperaturas observados. Assim consegue-se estimar os efeitos da variação climática na parte superior do poço. 


\section{Resultados}

O Perfil térmico do poço em Martins Farinheira serve como exemplo do procedimento adotado. O painel da esquerda da figura 6 ilustra a distribuição de temperaturas observadas. A linha pontilhada indica perfil condutivo para ajustes locais. As diferenças entre o perfil condutivo e os valores observados servem como valores residuais para tentativas de modelagem. O painel da direita da mesma figura ilustra o uso do procedimento de Bredehoeft e Papadopulos - BP em modelar a perturbação devida à infiltração de águas no solo. Neste painel, as temperaturas adimensionais, indicadas em cor vermelho, são caracterizados pelo gradiente térmico elevado e se posicionam ao longo da curva teórica para velocidade de infiltração de $0,0000001 \mathrm{~m} / \mathrm{s}$. As temperaturas adimensionais, em cor cinza, em profundidades menores, indicam valores estimados (e livre dos efeitos de perturbação climática), que se ajustam a curva teórica.

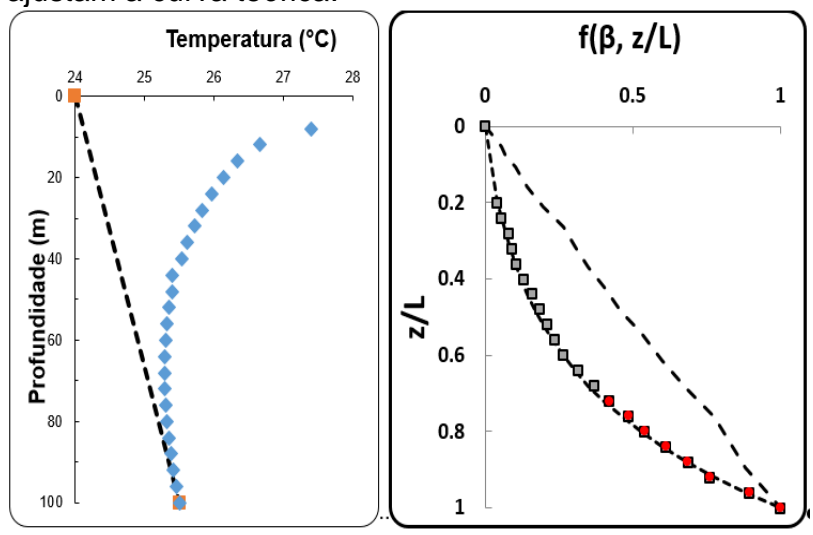

Figura 6 - Perfil de temperatura e distribuição de temperaturas numa camada permeável na presença de movimento vertical de fluidos

A diferença entre valores medidos e estimados indica efeitos de variação climática. O painel à esquerda da figura 7 ilustra ajuste ao modelo de variação climática sem correção. O painel à direita desta Figura indica ajuste aos dados corrigidos. Neste caso, admite-se a hipótese de que ocorreu aumento da temperatura da superfície de $2,3{ }^{\circ} \mathrm{C}$ nos últimos 30 anos.

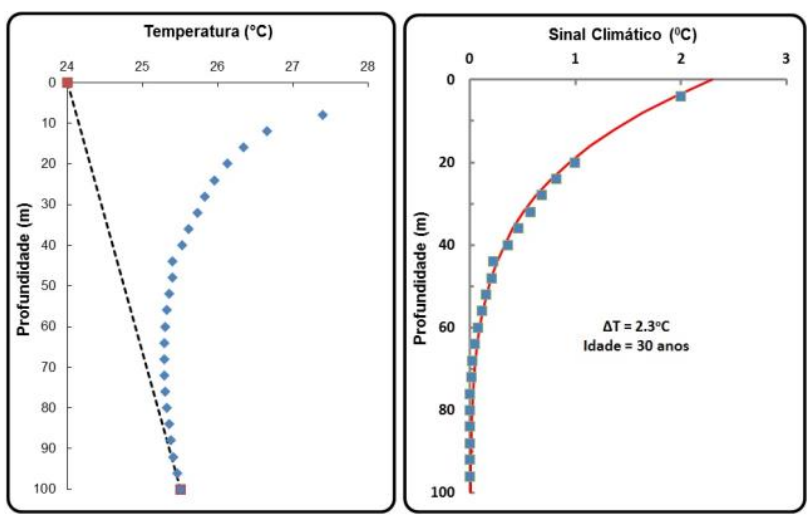

Figura 7 Modelo de ajuste adotado para partes rasas.
A profundidade do poço COPASA é de apenas 67 metros. A figura 8 indica o perfil e resultados de análise preliminar. Nota-se que as temperaturas são maiores do que obtidos no ajuste do modelo climático. Isso implica transporte de calor por movimento descendente de águas. Contudo, o analise do perfil não permitiu estimativas de velocidades de infiltração das águas.
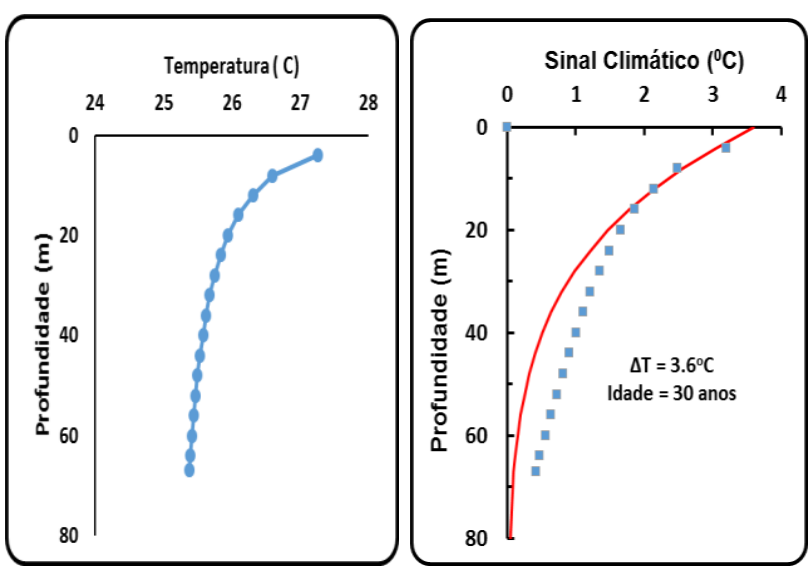

Figura 8. Perfil térmico do poço COPASA e ajuste do modelo climático. Nota-se que as temperaturas observadas são maiores que os do modelo climático, que é indicação de movimento descendente de águas.

Os perfis de poços João Soares e José João Pinheiro também apresentaram indícios de movimentos descendentes de águas. Contudo limitações de profundidade não permitiram estimativas de velocidades de fluxos descendentes de águas.

Apresentam-se na Tabela-1 as coordenadas de localização geográfica dos quatro poços perfilados e as faixas de profundidades pertinentes obtidas pelas perfilagens geotérmicas onde foram detectados movimentos de águas subterrâneas.

Tabela 1 - Sumário de resultados obtidos em poços perfilados indicando as profundidades $(P)$, velocidades de movimento de águas (V) perturbação climática (PC).

\begin{tabular}{|c|c|c|c|}
\hline Poço & $\mathrm{P}(\mathrm{m})$ & $\mathrm{V}(\mathrm{m} / \mathrm{s})$ & $\mathrm{PC}\left({ }^{\circ} \mathrm{C}\right)$ \\
\hline M. Farinheira & 96 & $3 \times 10^{-7}$ & 2.6 \\
\hline COPASA & 67 & $\approx 10^{-7}$ & 3.6 \\
\hline Joao Soares & 82 & $\approx 10^{-7}$ & $\approx 3$ \\
\hline J.J. Pinheiro & 52 & $\approx 10^{-7}$ & $\approx 3$ \\
\hline
\end{tabular}

\section{Discussão e Conclusões}

A análise dos dados e os resultados das perfilagens dos poços no presente trabalho permitiram as seguintes conclusões: foi constatada a existência de movimento descendente de águas nos quatro poços perfilados. As velocidades estão de ordem de $10^{-7} \mathrm{~m} / \mathrm{s}$. Também foram observados sinais de perturbação térmica induzida por mudanças climáticas das últimas décadas. Os ajustes de 
dados observados aos modelos unidimensionais de perturbações em poços rasos indicam ocorrências de mudanças nas temperaturas de superfície na faixa de 2 $3{ }^{\circ} \mathrm{C}$ nos últimos 30 anos.

\section{Agradecimentos}

Os autores desejam expressar seus agradecimentos ao Departamento de Geofísica do Observatório Nacional, pelo apoio institucional que tornou possível a realização deste trabalho.

\section{Referências}

ANA e Agência Nacional de Águas, 2003. PNRH e Plano Nacional de Recursos Hídricos 2003. Reference Document. Web site: http://www.ana.gov.br/pnrh/ documentos (accessed May 2003).

Birch, F., 1948. The effects of Pleistocene climatic variations upon geothermal gradients. Am. J. Sci. 246, $729 \mathrm{e} 760$.

Bredehoeft, J.D., Papadopulos, I.S., 1965. Rates of vertical groundwater movement estimated from the earth's thermal profile. Water Resour. Res. 1, 325e3

CPRM - Companhia de Pesquisa de Recursos Minerais. 2003. Mapa Geológico de Minas Gerais. Belo Horizonte: CPRM/COMIG. Escala 1: 1.000.000. Meio Digital.

CPRM - Companhia de Pesquisa de Recursos Minerais. 2005. Diagnóstico do município de Jenipapo de Minas, MG. Projeto Cadastro de Abastecimento por Águas Subterrâneas, Vale do Jequitinhonha.

Hamza, V.M., 1982, Pesquisas de medições de temperatura subsuperficial: Importância para a hidrogeologia. Revista de Águas Subterrâneas, v.5, p.65 - 99.

Hamza, V. M., (1991). Recent climate changes in the southern hemisphere: the geothermal evidence (In Portuguese), Proceedings 2nd Congress of the Brazilian Geophysical Society, Salvador (BA), 971-973.

Hamza, V.M., Muñoz, M., 1996. Heat Flow Map of South America, Geothermics, Vol. 25, nํ6, pg 599-646. Great Britain.

Hamza, V.M., Silva Dias, F.J.S., Gomes, A.J.L. and Terceros, Z.G.D., 2005. Numerical and Functional Representations of Regional Heat Flow in South America, PEPI, V. 152, 4, p.223-256.

Hamza, V.M., (2006). Thermal Signals of Global Warming: Evaluation using geothermal methods (in Portuguese), IV Symposium of the Amazon Region, 1214 September, Belém (PA), Brazil.

Hamza, V.M., (2007). Climate changes in the eastern segment of the Amazon region: Inferences based on geothermal methods (in Portuguese), V Symposium of the Amazon Region, 30 October to 1 November, Belém (PA), Brazil.

Hamza, V. M., Cavalcanti, A. S. B. and Benyosef, L.C., (2007). Surface thermal perturbations of the recent past at low latitudes-Inferences based on borehole temperature data from Eastern Brazil, Climates of the Past, 3, 1-1.

Mansure, A.J. and M. Reiter, (1979) A vertical ground water movement correction for heat flow. J. Geophys. Res. V. 84, PP. 3490-3496.

Ribeiro, F., (1991). Reconstruction of the climate change from geothermal profiles: Advances in Theory and Practice (in Portuguese), Proceedings 2nd Congress of the Brazilian Geophysical Society, Salvador (BA), 959964.

Souza, J. R. S., Araújo, R. L. C. and Makino, M., (1991). Transient variations in shallow thermal profiles associated with microclimatic changes in the Amazon (in Portuguese), Proceedings 2nd Congress of the Brazilian Geophysical Society, Salvador (BA), 965-970.

Stallman, R.W., 1963, Computation of groundwater velocity from temperature data. U.S. Geol. Survey Water Supply Paper 1544-H: 36-46.

Turner, F. J. \& Werhoogen, J., 1960. Igneous and metamorphic petrology. 2nd ed., McGraw Hill, New York, USA. 